\title{
Fetal brain issues in congenital heart disease
}

\author{
Fu-Tsuen Lee ${ }^{1,2}$, Mike Seed $^{2,3}$, Liqun Sun $^{2}$, Davide Marini ${ }^{2}$ \\ ${ }^{1}$ Department of Physiology, Faculty of Medicine, University of Toronto, Toronto, Canada; ${ }^{2}$ Division of Cardiology, Department of Paediatrics, \\ Hospital for Sick Children, University of Toronto, Toronto, Canada; ${ }^{3}$ Department of Diagnostic Imaging, Hospital for Sick Children, University of \\ Toronto, Toronto, Canada \\ Contributions: (I) Conception and design: All authors; (II) Administrative support: None; (III) Provision of study materials or patients: None; (IV) \\ Collection and assembly of data: FT Lee; (V) Data analysis and interpretation: FT Lee; (VI) Manuscript writing: All authors; (VII) Final approval of \\ manuscript: All authors. \\ Correspondence to: Davide Marini, MD, PhD; Liqun Sun, MD, PhD. Division of Cardiology, Department of Paediatrics, Hospital for Sick Children, \\ University of Toronto, 555 University Avenue, Toronto, Ontario M5G 1X8, Canada. Email: davide.marini@sickkids.ca; liqun.sun@sickkids.ca.
}

\begin{abstract}
Following the improvements in the clinical management of patients with congenital heart disease (CHD) and their increased survival, neurodevelopmental outcome has become an emerging priority in pediatric cardiology. Large-scale efforts have been made to protect the brain during the postnatal, surgical, and postoperative period; however, the presence of brain immaturity and injury at birth suggests in utero and peripartum disturbances. Over the past decade, there has been considerable interest and investigations on fetal brain growth in the setting of CHD. Advancements in fetal brain imaging have identified abnormal brain development in fetuses with CHD from the macrostructural (brain volumes and cortical folding) down to the microstructural (biochemistry and water diffusivity) scale, with more severe forms of CHD showing worse disturbances and brain abnormalities starting as early as the first trimester. Anomalies in common genetic developmental pathways and diminished cerebral substrate delivery secondary to altered cardiovascular physiology are the forefront hypotheses, but other factors such as impaired placental function and maternal psychological stress have surfaced as important contributors to fetal brain immaturity in CHD. The characterization and timing of fetal brain disturbances and their associated mechanisms are important steps for determining preventative prenatal interventions, which may provide a stronger foundation for the developing brain during childhood.
\end{abstract}

Keywords: Fetal; brain; congenital heart disease (CHD); imaging

Submitted Aug 04, 2020. Accepted for publication Aug 27, 2020.

doi: $10.21037 / \mathrm{tp}-20-224$

View this article at: http://dx.doi.org/10.21037/tp-20-224

\section{Introduction}

Congenital heart disease (CHD) is the most common type of birth defect, affecting 7 to 9 per 1,000 newborns (1). Over the past several decades, the focus on improving cardiac surgical techniques and intensive care has resulted in a significant decrease in mortality even for the most severe forms of CHD (2). With the increasing number of children achieving successful cardiac repairs and reaching adulthood, observations on neurodevelopmental deficits have surfaced in these survivors. Neurodevelopmental delay is now considered the most important comorbidity in patients with CHD and covers deficits in cognition, behaviour, and motor skills (3). These impairments may be recognized in patients with a wide range of cardiac defects, from simple to the most complex CHDs. Typically, these neurodevelopmental deficits appear during childhood and persist until adulthood, ultimately affecting societal contribution and quality of life. This results in a lifelong need for remedial services and is becoming a considerable burden in terms of cumulative cost for society $(4,5)$.

Strategies aimed to improve intraoperative management and intensive care have been attempted to minimize neurological insult during the neonatal and infant period. However, patient-specific factors such as genetic makeup, CHD pathophysiology, and perinatal history are now 


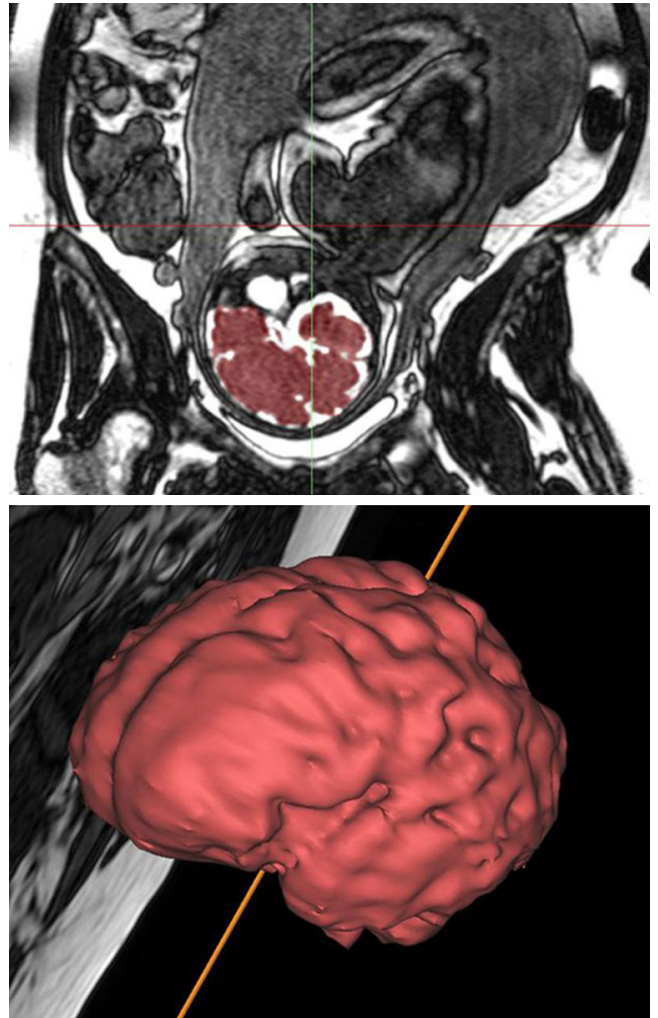

Figure 1 Fetal brain volume obtained from segmentation of the three-dimensional steady-state free precession acquisition by MRI [image adapted from reference (16)]. MRI, magnetic resonance imaging.

explored with increased interest as key determinants for neurodevelopmental outcomes (6). The presence of brain immaturity (7-10) and injuries (11) identified by magnetic resonance imaging (MRI) even before surgery suggests disturbances of brain growth during the fetal period. In parallel, the advancements in fetal imaging have provided exciting tools capable of (I) measuring specific fetal brain properties such as brain volumes, cortical folding, biochemistry, water diffusivity, and tissue oxygenation and (II) describing the cardiovascular pathophysiology and quantifying parameters impacting brain growth and maturation such as cerebral oxygen delivery, consumption, and extraction. This article will review the up-to-date techniques used to assess fetal brain development, the main brain issues found in fetuses with CHD from the macro- to microscopic structural level, the potential neurobiological mechanisms of origin, and the clinical consequences transpiring after birth.

\section{Advancements in fetal brain imaging}

Historical clinical observations at birth of infants with CHD have highlighted patterns of fetal growth differences when compared to their healthy counterparts. For example, infants with transposition of the great arteries (TGA) have a relatively smaller head to normal birth weight, infants with tetralogy of Fallot (TOF) are smaller in all dimensions, and infants with hypoplastic left heart syndrome (HLHS) are smaller in all dimensions with a relatively smaller head (12). Fast forward, current fetal imaging technology has the ability to safely observe through the maternal abdominal wall to directly assess fetal brain growth during pregnancy (13). Clinically, ultrasonography (US) is the primary imaging method for routine evaluation of the fetal brain during pregnancy as it is praised for its accessibility and ease of use (14). However, US image quality may be significantly limited by maternal obesity, oligohydramnios, and the fetal position (13).

Fetal MRI has become of increasing importance as an adjunct to prenatal US. MRI is the ideal technique to image the brain when compared to US because of its superiority to contrast soft tissue and fluids and great resolution to detect subtle structural alterations and injuries (15). MRI is more accurate in measuring total and regional volumes of the fetal brain (Figure 1) and has proved to have higher sensitivity in detecting intracranial structural abnormalities in fetuses with CHD (17). However, fetal MRI has been plagued with long scan times, movement artifacts, and accessibility issues. Throughout the past two decades, improvements in fetal MRI methodology such as fast imaging sequences and motion correction techniques have increased image quality and accuracy (15), allowing the visualization of the properties of fetal brain tissue from the macrostructural to microstructural level $(18,19)$.

\section{Macrostructural fetal brain abnormalities}

\section{Total and regional brain size}

The first landmark study on in utero brain disturbances in CHD demonstrated progressively abnormal brain measures starting in the third trimester when compared to healthy controls (Figure 2) (20). From this point forward, there has been a substantial amount of investigation to establish the precise timing and patterns of delay in fetal brain growth in fetuses with CHD through US and MRI. Fetal brain volumes acquired by MRI have homogeneously 
confirmed fetal brain growth deficits during the third trimester both in fetuses with heterogeneous isolated CHD (16,17,20-22) and in homogeneous critical CHDs such as TGA (23), TOF (24), and left-sided CHD $(25,26)$. These observed reductions in volumetric brain growth coincides with the critical window of accelerated fetal brain growth (27), which has led to the interpretation of diminished cerebral substrate delivery (mainly oxygen and glucose) secondary to altered fetal cardiovascular physiology may be unable to match the increasing cerebral metabolic demand during the third trimester. However, the onset of reduced volumetric brain growth is not limited to the third trimester as many studies have also found reductions in fetal head biometry beginning in the second trimester in both major and minor CHD (28-32), though more prominent in fetuses with single ventricle physiology (SVP), HLHS, TOF, and TGA. More compellingly, fetuses with CHD, especially cyanotic subtypes, have demonstrated abnormal fetal head biometry in the first trimester (33) and as early as 11-14 GW in fetuses with left heart hypoplasia (34), suggesting a potential genetic contribution towards abnormal brain development in CHD.

To further explore the peculiarities of the fetal brain in CHD, the growth of specific regions and structures of the brain has been subsequently studied. Using MRI, a progressive decline in the volumetric growth of cortical gray, subcortical gray, and white matter has been demonstrated in fetuses with HLHS during the third trimester (35) and TOF (24) during the second trimester. When the fetal brain is subdivided into its three main structures: the cerebrum, cerebellum, and brainstem, one study found fetuses with CHD have reduced cerebral but larger brainstem volumes whereas cerebellar volumes were not reduced despite an overall reduction in total brain volume (21). Since the brainstem is involved in basic body functions essential for life such as respiratory and cardiac regulation, it might be possible that the fetal brain privileges the growth of more indispensable structures at the expense of others in the setting of CHD. Fetal lamb studies have demonstrated a hierarchical flow redistribution in response to hypoxemia that prioritizes the brain stem followed by the cerebellum and then the cortex $(36,37)$. The preservation of cerebellar volume was also observed in fetuses with TGA (23) and TOF (24) compared to their healthy counterparts. However, other studies have found reductions in cerebellar volume in cyanotic and severe forms of CHD (38-40), suggesting that these fetuses may not fully compensate for altered hemodynamics as the cerebellum is extremely vulnerable to hypoxia (41) or that there may be additional mechanisms involved, such as genetic predisposition. Other structures possibly affected are the frontal lobes and thalamus, which have been found to be reduced in volume starting in the third trimester. The largest reductions were associated with diagnostic category, the worst being HLHS followed by aortic hypoplasia, TGA, and TOF (39).

In fetuses with critical CHD, fetal brain volumes are strongly related to preoperative brain volumes (42). Furthermore, smaller fetal brain volumes in the third trimester are linked to an increased risk for acquired neonatal ischemic brain injury (42). This recent finding suggests that delayed brain growth and maturation may predispose the brain to increased vulnerability to disturbances in cerebral blood flow potentially encountered during the transition from fetal to neonatal life, preoperative management, cardiac surgery, and postoperative intensive care. Ischemic brain injuries causing neuronal death and Wallerian degeneration result in further brain volume loss, with severe cases reaching diffuse cerebral atrophy.

\section{Cortical folding and other abnormal structural brain findings}

The advancements in MRI has enabled 3-D reconstruction of the fetal cortex with algorithms measuring cortical surface area and cortical depth $(35,43)$. In the normal fetal brain development, the formation of gyri ("bumps") and sulci ("grooves") begins at the 8th week of gestation. This process is called cortical folding and is believed to allow the cerebral cortex to attain a large surface area to fit in a relatively small cranial volume $(27,44,45)$.

Recent imaging studies of fetuses with CHD have shown alterations in cortical patterning and folding beginning at mid-gestation. Neuroanatomical landmarks for sulciknown as sulcal basins-have been observed to have a different spatial distribution in fetuses with CHD as early as $22 \mathrm{GW}$ when compared to healthy controls (46). This is followed by a reduction in cortical folding and surface area that has been reported as early as 25 weeks but most prominent during the third trimester, reflected by shallower sulci and fissure depth, more symmetric brains, and delayed opercular development $(22,35,46,47)$. Clinically, an open operculum is recognized as a sign of brain immaturity and found in $17-90 \%$ of term infants with complex CHD having preoperative MRI $(7,48)$.

Alongside impaired brain parenchymal development, 

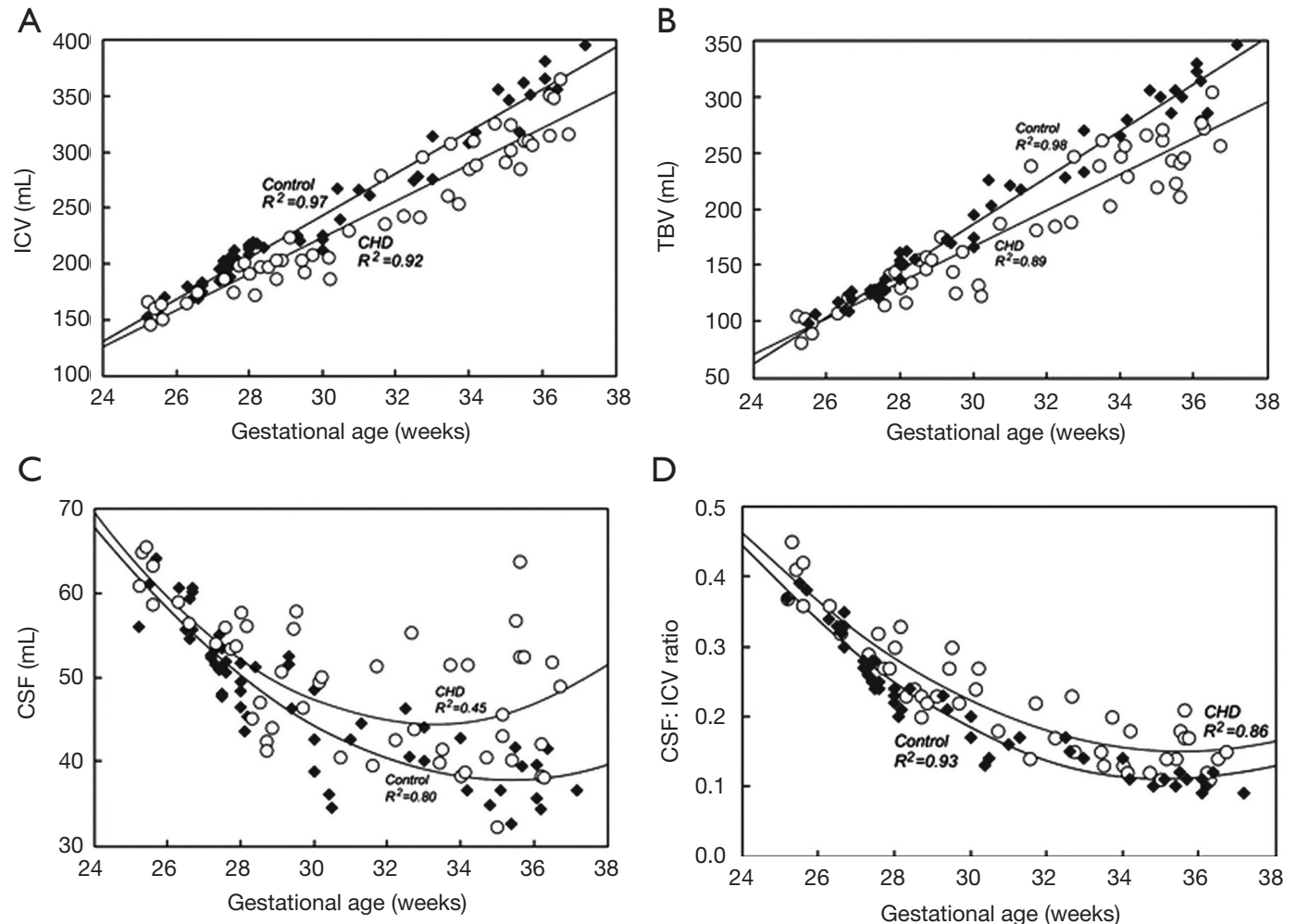

Figure 2 Progressively abnormal brain measures in fetuses with CHD starting at the beginning of the third trimester by MRI [image adapted from reference (20)]. CHD, congenital heart disease; MRI, magnetic resonance imaging; ICV, intracranial cavity volume; TBV, total brain volume; CSF, cerebrospinal fluid.

evidence of impaired cerebrospinal fluid (CSF) development and regulation has been observed in isolated CHD during the fetal period. Fetuses with more severe forms of CHD have demonstrated increased CSF spaces and volumes as early as $20 \mathrm{GW}$ and persisting until term assessed by MRI $(20,23,24,42,49,50)$. During brain development, CSF serves to support fetal brain growth by delivering growth factors, signalling molecules, and nutrients and provides hydrostatic pressure to the expanding brain (51). Larger CSF volumes have been hypothesized as an initial response to sustain the growth of the fetal cranium during parenchymal brain underdevelopment or a disturbance in CSF dynamics secondary to altered fetal cardiovascular physiology, or both $(24,50)$. However, despite a compensatory increase in CSF spaces, fetuses and newborns with CHD still have smaller head circumferences and intracranial cavity volumes than normal controls $(20,24,42,50)$. Interestingly, enlarged CSF spaces have been associated with poorer neurodevelopmental outcomes in neonates with single ventricle physiology (52).

Other CSF related abnormalities occasionally found in fetuses with CHD during the second and third trimesters include asymmetrical ventricles, ventriculomegaly, and ventricular cysts $(42,49,50)$. Ventriculomegaly is the most common fetal brain abnormality found in CHD (53), and its pathogenesis has been described as the result of developmental malformations or cerebral atrophy due to insufficient cerebral substrate delivery (49). Moreover, animals exposed to a chronic substrate deficiency had a reduction in brain size and developed ventriculomegaly (54).

More severe reduction in brain volumes such as microcephaly (head circumference $\leq 3 \mathrm{rd}$ percentile) are also associated with isolated CHD and typically results in cognitive dysfunction $(55,56)$. In cases of isolated HLHS, the prevalence of microcephaly at birth full-term is $12 \%$ $(57,58)$, whereas cases of HLHS associated with other malformations are $45 \%$ at autopsy (59).

Other clinically relevant abnormalities such as vermian hypoplasia, enlarged cisterna magna, and agenesis of the corpus callosum have been noted using fetal MRI in fetuses with CHD without genetic syndromes $(17,21,49,50,53)$. Overall, CHD patients with these structural abnormalities 

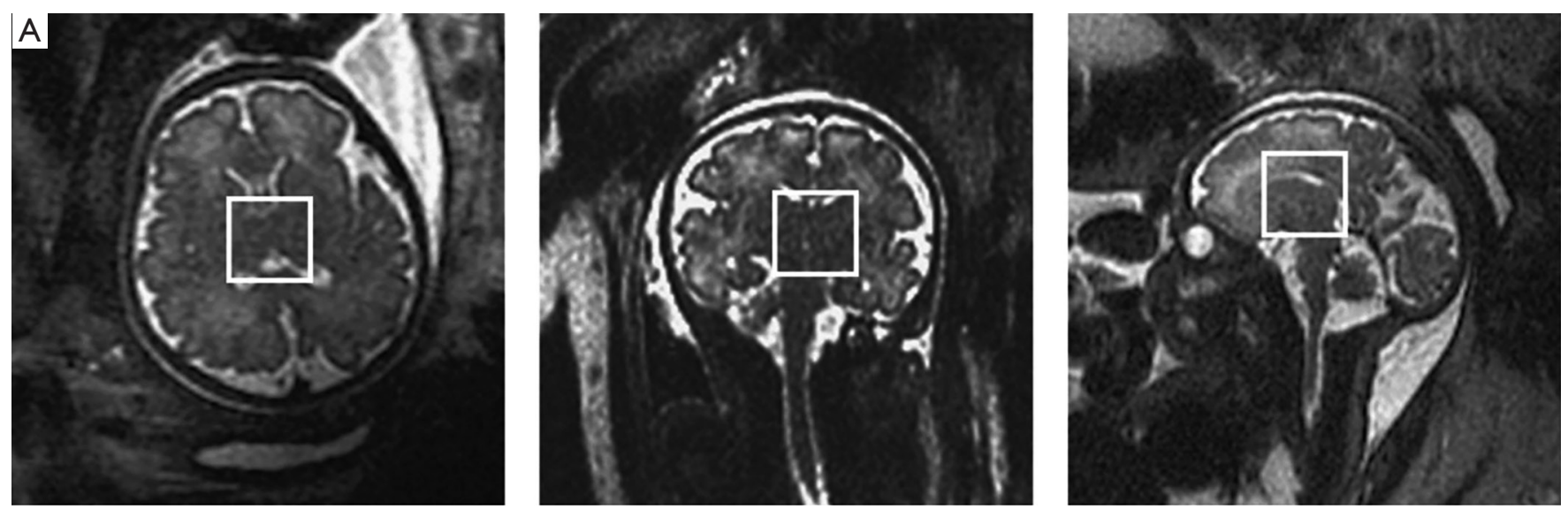

B

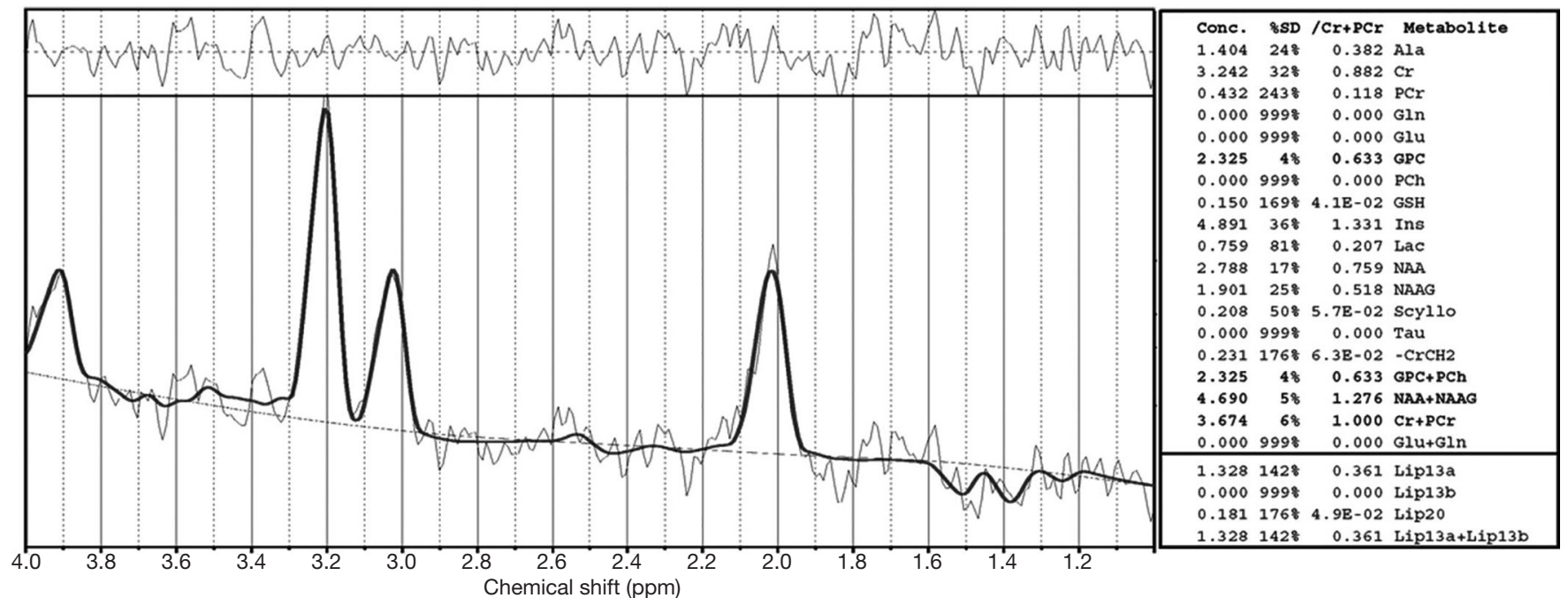

Figure 3 MRS acquisition showing (A) voxel placement in the axial, coronal, and sagittal planes and the (B) different chemical peaks of the selected fetal brain tissue [image adapted from reference (60)]. MRS, magnetic resonance spectroscopy.

present a variable neurodevelopmental phenotype after birth ranging from normal to severe neurodevelopmental impairment.

\section{Microstructural abnormalities of the fetal brain in CHD}

\section{Magnetic resonance spectroscopy (MRS)}

Fetal brain MRS provides a non-invasive technique to measure cerebral metabolites (Figure 3). Changes in fetal brain metabolic concentrations in the setting of CHD may indicate in utero disturbances of brain development, and thus, they could potentially represent useful predictive biomarkers of neurodevelopmental impairment (60).
However, fetal MRS may be prone to sampling errors due to movement artifacts. In the event metabolic markers normally present in the CSF are sampled as brain tissue, this may lead to a pathologic interpretation of brain dysmaturation if detected in brain tissue (60).

Studies using MRS have revealed abnormal cerebral metabolism in fetuses with CHD. A slower rate of increase in $\mathrm{N}$-acetyl aspartate (NAA) to choline (Cho) ratio (20) has been demonstrated, indicating a relative reduction in dendritic and synaptic development over gestation (20). Furthermore, cerebral lactate, which indicates a shift from aerobic to anaerobic metabolism possibly due to impaired cerebral oxygen delivery, was found in fetuses with diminished antegrade aortic flow (20). These results were also reproduced in another study whereby NAA: Cho 
decreased in the frontal area and basal ganglia whereas inositol (Ino): Cho increased (22). Increased Ino is a marker of astrocyte activation and reactive gliosis which has been suggested to represent a response to neural injury or hypoxic conditions aimed to maintain neurogenesis (61). Although increased Ino has been associated with increased lactate (62), only 3 of 48 fetuses with CHD assessed with MRS in this study had identified cerebral lactate (22). Currently, the presence of lactate as a biomarker for abnormal fetal brain development remains controversial since it has also been detected in healthy fetuses, suggesting that lactate might be an important source of energy for brain growth and maturation and part of its normal metabolic spectrum $(19,60)$.

\section{Diffusion tensor imaging (DTI)}

Fetal magnetic resonance DTI is a relatively new technology used to image white matter microstructure of the brain and assess its maturation. DTI measures the diffusion of water by two main patterns: water diffusing in all directions (isotropic diffusion) and along one direction (anisotropic diffusion). In myelinated white matter, water tends to preferentially diffuse along the axonal tract axis (axial diffusivity) rather than perpendicular (radial diffusivity) across the axonal membrane and sheath towards the extracellular space. Therefore, as the brain matures, progressive myelination of axons increases the anisotropic environment with a diffusion maximum parallel to the orientation of the fibre tracts while restricting diffusion perpendicular to the axonal tracts (63). Recently, DTI performed on fetuses with CHD have demonstrated decreased axial diffusion and increased radial diffusion in the corpus callosum when compared to healthy controls from 26 to $30 \mathrm{GW}$ (64). This evidence supports the hypothesis that the white matter immaturity detected by DTI in newborns with CHD may originate during the prenatal period $(8,11,65)$. Furthermore, brain immaturity appears to play a role in the high prevalence of postnatal hypoxic-ischemic white matter injury and periventricular leukomalacia seen in CHD (66), which have been associated with worse cognitive and motor outcomes later in childhood life $(67,68)$.

\section{Factors affecting fetal brain growth in CHD}

\section{Genes affecting the heart and brain}

Currently, approximately $20 \%$ of CHD cases are associated with genetic or chromosomal abnormalities, many of which have co-existing neurodevelopmental deficits $(69,70)$. Genetic syndromes such as Down, Williams, Alagille, Noonan, DiGeorge (22q11.2 microdeletion) syndromes, among others have a high prevalence of CHD and neurodevelopmental delay occurring together, suggesting common underlying genetic pathways that may account for this phenotype (71).

The first indication of genetic contributions on neurodevelopmental delay in the setting of nonsyndromic CHD started with identifying the APOE gene $\varepsilon 2$ allele that was associated with worse neurodevelopmental outcomes after cardiac surgery (72). This important finding demonstrated that certain genetic polymorphisms were associated with impaired neuroresiliency and neuronal repair capacity after surgery-related insults. One decade later, pathogenic copy number variants were found to be linked to single ventricle forms of CHD with intellectual disabilities (73). In this study, single ventricle patients with neurocognitive and growth deficits had a significantly higher incidence $(13.9 \%)$ of pathogenic copy number variants than those without (4.4\%) (73).

More recently, exome sequencing of CHD parentoffspring trios revealed an excess of de novo mutations. These mutations accounted for $20 \%$ of patients with CHD, neurodevelopmental delay, and extracardiac abnormality, but only $2 \%$ with isolated CHD. Since the mutations occurred in genes highly expressed both in developing heart and brain, it is possible that not only the CHD but also the neurodevelopmental delay and/or the other extracardiac congenital anomalies could be caused by the same de novo mutations, and part of the phenotype (74). Interestingly, many of these mutated genes were associated with chromatin-modifying pathways, suggesting an impairment in epigenetic regulation $(74,75)$. Based on the current understanding, polygenic inheritance and/or multifactorial etiology are likely to play a crucial role in the association between neurodevelopmental delay and CHD.

\section{Impaired cerebral substrate delivery and brain sparing response in $\mathrm{CHD}$}

Fetal circulations in normal fetuses and those with complex CHD have been described by using cardiac MRI (Figure 4). In the normal fetal circulation, well-oxygenated blood returning from the placenta bypasses the liver through the ductus venosus and streams up the inferior vena cava, separately from the desaturated systemic venous blood returning from the lower limbs and visceral organs. At the 

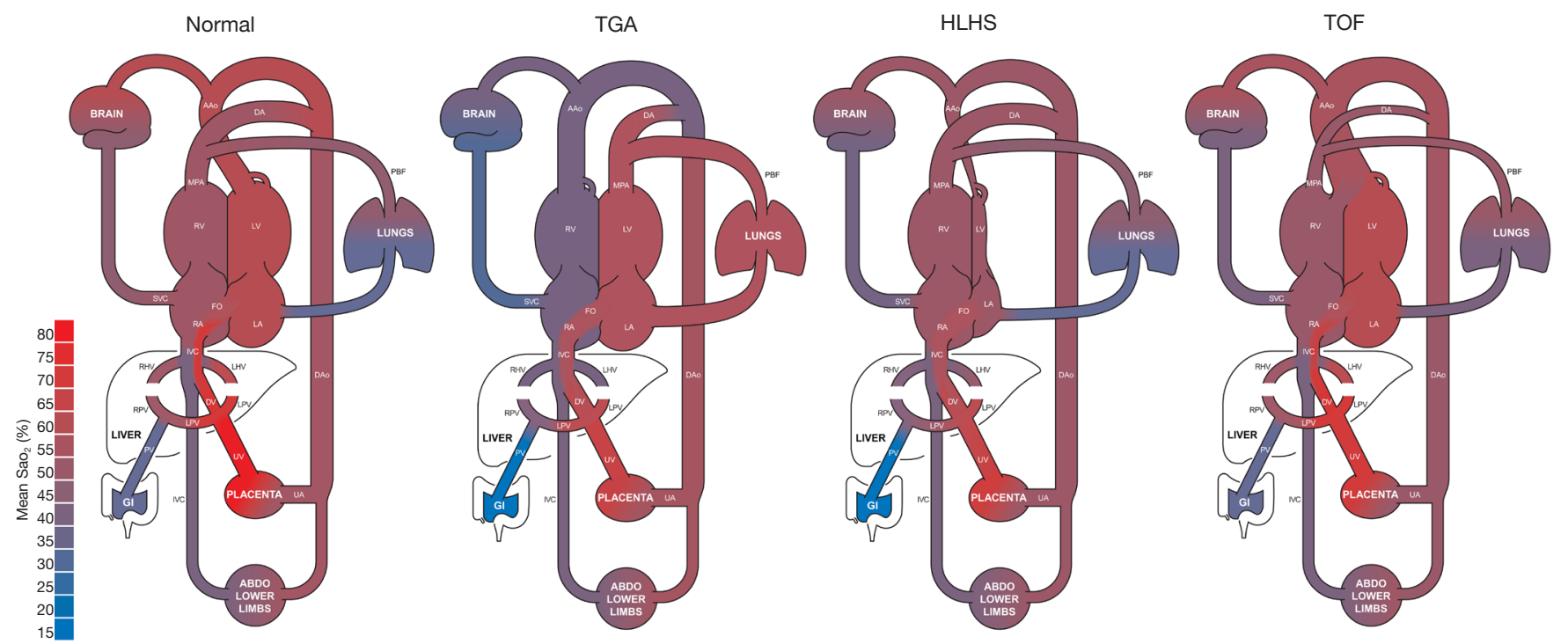

Figure 4 Representations of fetal circulations in normal, TGA, HLHS, and TOF by MRI [image adapted from reference (16)]. TGA, the great arteries; HLHS, hypoplastic left heart syndrome; TOF, tetralogy of Fallot; MRI, magnetic resonance imaging.

junction of the inferior vena cava and the right atrium, the Eustachian valve redirects the well-oxygenated placental blood across the foramen ovale into the left atrium. This mechanism, known as fetal cardiac streaming, results in higher oxygen saturations in the ascending aorta (AAo) than the main pulmonary artery (MPA). This preferential streaming provides the brain - the most metabolically active fetal organ-most of the oxygenated blood to support fetal brain development (76). In addition, other substrates such as glucose freshly transferred from the placenta are also preferentially streamed towards the brain (77). By contrast, fetuses with CHD have altered cardiac streaming such that substrate delivery to the brain is disrupted, leading to a cerebral metabolic supply-demand mismatch. Cerebral arterial vessels are equipped with an effective autoregulatory mechanism in which under a substrate deficient setting will vasodilate to increase cerebral blood flow to match cerebral metabolic demand $(76,78)$. This mechanism is known as brain sparing physiology; however, may not be adequate for severe or chronic circulatory perturbations in certain forms of CHD.

Many investigations have attempted to describe the timing and the protective effect of brain sparing and the inadequate responses during fetal brain development in different forms of CHD. The pulsatility index (PI) measured by Doppler US is the key parameter for assessing vascular resistance and is calculated as:

$$
\mathrm{PI}=(\mathrm{PSV}-\mathrm{EDV}) / \mathrm{TAV}
$$

Where PSV is the peak systolic velocity, EDV is the enddiastolic velocity, and TAV is the time-averaged (mean) velocity. For this purpose, the middle cerebral artery (MCA) has been consistently used as the vessel of choice for its accessibility and sensitivity to hypoxemia (79).

In healthy fetuses, the MCA-PI decreases (i.e., vasodilates) at around $30 \mathrm{GW}$ until term presumably to permit increased blood flow towards the brain to facilitate the increased metabolic demand of the fetal brain required during a period of accelerated brain development (80). However, fetuses with CHD have a more pronounced decrease in MCA-PI when assessed after the late-second trimester $(22,47,81-84)$. This has been hypothesized as a mechanism that serves to increase cerebral blood flow to match cerebral metabolic supply to demand.

In HLHS, the left-sided heart structures are severely malformed, resulting in the mixing of systemic and pulmonary venous return in the right side of the heart. In the case of aortic and/or mitral atresia, the right ventricle conducts the entire combined ventricular output through the MPA and sustains the systemic circulation through the ductus arteriosus (76). Therefore, the upper extremities, including the brain, are supplied via retrograde flow across the aortic isthmus. In the case of retrograde coarctation or transverse arch hypoplasia, restriction of the blood flow to 
the brain may occur (81). By the time the blood reaches the brain, the blood flow and substrate content are reduced, such that there is a profound decrease in cerebrovascular resistance in an attempt to attract blood flow to match the metabolic demands of the brain. Fetuses with HLHS have been described to have earlier and larger reductions in MCA-PI compared to other forms of CHD (85-88), and retrograde flow at the distal arch has been demonstrated to elicit the brain sparing response (87). The absence of antegrade flow in the AAo has been associated with decreased white matter, subcortical gray matter volumes, and surface area of the right hemisphere in fetuses with HLHS compared to normal controls (35). However, normal head growth in HLHS fetuses with aortic atresia may suggest that in the absence of retrograde coarctation or transverse arch hypoplasia, the brain sparring response may be adequate for brain growth (31). On the contrary, HLHS fetuses with normal late-gestational MCA-PI dopplers but head growth abnormalities may suggest that normalization of cerebral blood flow occurs as a consequence of cerebral metabolic suppression in the setting of deficient substrate supply, as explained later. In less severe forms of left-sided CHD such as mild to moderate left ventricular outflow tract obstruction, because of the antegrade flow in the AAo, autoregulatory compensation mechanisms are less marked than in HLHS, but still present to satisfy the metabolic demand of the brain $(81,85)$. To note, in contrast to the previous studies, an inverse correlation was found between AAo diameter and MCA-PI in fetuses with HLHS and small brain volume (89). In this study, cerebrovascular resistances were higher in those fetuses with smaller AAo. This finding was hypothesized to be due to the underdevelopment of the cerebral vascular bed as revealed by large brain capillaries, low capillary density, and abnormal molecular markers in the germinal matrix in specimens of terminated fetuses, explaining the elevated cerebrovascular resistance and the lack of brain sparing effect (89).

In TOF, cases with pulmonary valve stenosis have a variable proportion of blood shunted from right-to-left across the ventricular septal defect into the AAo (76). The intra-cardiac mixed blood resulting in relatively substrate deficient blood delivered to the brain would be expected to cause cerebral vasodilatation. However, the increased aortic output, on the contrary, may trigger a protective brain-sparring mechanism by cerebral vasoconstriction (24). In more severe cases such as TOF with pulmonary atresia, there is complete intracardiac mixing and the AAo conducts the entire cardiac output (76). In this scenario, the profound increase in aortic flow may cause a considerable cerebral vasoconstrictive response that outweighs the expected vasodilatory response aimed to increase cerebral substrate delivery. The balance between the autoregulatory response to substrate content and cerebral perfusion in this heterogeneous group of right outflow tract obstruction could explain why in most of the studies, MCA-PI is found increased in fetuses with pulmonary atresia but often normal in fetuses with pulmonary stenosis $(85-87,90,91)$.

In TGA, the MPA arises from the left ventricle and the AAo arises from the right ventricle. As a result, substrate rich blood from the placenta travels to the pulmonary vasculature and lower extremities via the ductus arteriosus. On the contrary, the brain is perfused by the substrate depleted blood returning from the caval veins and is expected to elicit a brain sparing response (76). Reductions in MCA-PI have been demonstrated in fetuses with TGA (92); however, other studies have found no difference when compared to their healthy counterparts $(86,87)$. One potential explanation is that half of the fetuses with TGA had a ventricular septal defect, which is thought to cause bidirectional shunting in utero. During systole, there is right-to-left shunting, whereas at diastole, there is left-toright shunting and thus, improving the substrate content of the blood flow supplying the brain (76).

The relationship between brain sparing and cerebral growth is not fully understood. Fetuses with CHD have demonstrated to have an inadequate increase in both fetal head circumference and brain volume despite low MCAPI, suggesting that brain sparing response might not be sufficient to guarantee normal cerebral growth (30).

The inconsistent findings of the association between MCA-PI and fetal brain growth may be related to the metabolic demand and timing of the investigation. Four patterns of relationship between substrate delivery and consumption to fetal brain development may be hypothesized in the setting of CHD:

(I) Cerebral substrate delivery satisfies cerebral metabolic demand to facilitate normal brain growth;

(II) A decrease in MCA-PI may provide enough flow compensation to satisfy cerebral metabolic demand to facilitate normal brain growth;

(III) A decrease in MCA-PI may not provide enough flow compensation to satisfy cerebral metabolic demand resulting in abnormal brain growth;

(IV) No apparent change in MCA-PI because of chronic 


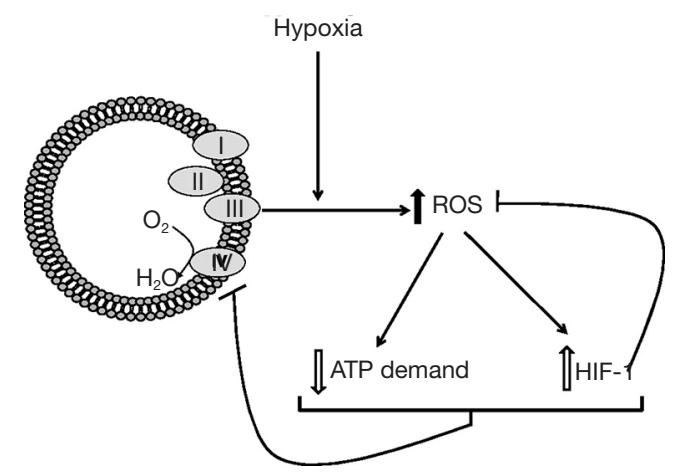

Figure 5 A diagram of the negative feedback cycle between hypoxia and the rate of mitochondrial respiration. Hypoxia increases reactive oxygen species (ROS) production from mitochondrial complex III. Multiple molecular pathways respond and decrease ATP utilization, resulting in a decrease in the mitochondrial respiration rate. In tandem with the hypoxia-inducible factor pathway, ROS production decreases, thereby preventing cell damage and death [image adapted from reference (95)].

exposure to substrate deficiency may be the result of the brain downregulating its metabolic demand to avoid anoxia-induced cell injury, resulting in abnormal brain growth.

Failure to adapt to inadequate substrate delivery resulting in brain dysmaturation and reduced growth is hypothesized to occur in the third trimester, a critical window of accelerated brain development normally characterized by extensive axonal outgrowth, dendritic expansion, synaptogenesis, and glial proliferation leading the cortical volume to increase by 4 -fold (27). In an animal hypoxia model, it has been demonstrated that oligodendrocyte precursor cells prioritize angiogenesis to alleviate the hypoxic conditions rather than differentiating into myelinating oligodendrocytes (93). Fetal sheep and guinea pig in a chronic substrate deficient setting have shown that reduced brain growth is mediated by reduced myelination and synaptogenesis (54). This potential mechanism may account for white matter dysmaturation observed in fetuses with CHD. In one study, neuropathological findings on eleven electively aborted HLHS fetuses all demonstrated varying degrees of white matter injury and increased GFAP and CD68 expression in brain tissue, markers of chronic white matter injury (31).

Recent advancements in fetal cardiac magnetic resonance imaging have provided a novel non-invasive approach to measuring oxygen delivery and consumption of both the fetal body and the brain by using a combination of cine phase-contrast MRI with metric optimized gating and T1 and T2 mapping (94). When coupled with fetal MRI brain volumetry, this has revealed fetuses with CHD have a reduction in fetal cerebral oxygen consumption, which was associated with smaller fetal brain size (16). This evidence supports the hypothesis that the fetal brain may downregulate cellular metabolism in the setting of a chronic reduction in cerebral oxygen delivery with a consequence of delayed growth. Biochemical studies have demonstrated under chronic hypoxic conditions, cells shift into a state of metabolic suppression by inhibiting non-essential metabolic activities and preserve the limited energy production for fundamental functions necessary for cell survival (Figure 5) (95). Similarly, metabolic suppression is observed during myocardial hypoxia resulting in decreased contractile activity, known as myocardial hibernation (95). Therefore, chronic hypoxia secondary to altered hemodynamics due to cardiac malformations could possibly further depress fetal cardiac output and compound the decrease in cerebral substrate delivery with further potential implications for fetal brain development. More recently, fetal brain MRI using transversal relaxation time $\left(\mathrm{T}^{*}\right)$, which depends on the tissue concentration of deoxyhemoglobin, has led to a demonstration of reduced cerebral tissue oxygenation in fetuses with major CHD compared to fetuses without CHD $(96,97)$.

Although the role of oxygen has been the most extensively investigated, the existence and contributions of other important substrates should not be underestimated. Abraham Rudolph has raised the hypothesis that cerebral developmental impairment may be more related to inadequate glucose rather than oxygen supply, based on the observation of robust cerebrovascular responses in animal hypoxia models and the lack of elevated lactate in the majority of fetuses with CHD (77). The development of technology capable to non-invasively quantify fetal blood glucose levels will potentially allow the investigation of this hypothesis in the future.

\section{The placenta and the brain}

As the only communicating organ between the mother and fetus, the placenta fundamentally dictates fetal growth such that proper placental development throughout gestation is critical for the normal course of fetal development (98). In fact, the placenta and fetal heart 
develop in parallel in a synergistic pattern such that it has been referred to as the heart-placental axis (99). Both organs share several developmental pathways that point to a potential common vulnerability to genetic defects (98). Moreover, abnormal fetal cardiovascular circulation may result in acquired placental lesions. Placentas of newborns with CHD have been found to be smaller than expected and manifest a number of vascular abnormalities such as chorangiosis, hypomature villi, thrombosis, and infarction (100). Furthermore, fetal thrombotic vasculopathy was recently associated with a 6 -fold increase in cardiac abnormalities (101), potentially leading to a further acquired impairment in placental function.

Abnormal placental function has also been implicated as an important contributor to the abnormal fetal brain development seen in fetuses with CHD. In fetuses with HLHS, gross placental evaluation has revealed scarce villus expansion during the third trimester and reduced placental weights (102). This evidence supports the hypothesis that abnormal fetal brain development and reduced somatic growth in these fetuses might be mediated, in conjunction with other factors, by a mismatch between the reduced surface area available for nutrient and oxygen exchange and the amount required in a period normally characterized by the exponential brain and somatic growth of the fetus. As a surrogate to account for placental function during the US assessment for the brain sparing response, the cerebroplacental ratio (CPR) is normally used and can be calculated as:

\section{$\mathrm{CPR}=\mathrm{MCA}-\mathrm{PI} / \mathrm{UA}-\mathrm{PI}$}

Where the CPR is the ratio between the pulsatility indexes in MCA (MCA-PI) and umbilical artery (UA-PI), respectively.

According to this formula, fetuses with severe forms of CHD with healthy placental function are expected to have low CPR because the MCA-PI decreases as a brain sparing response to diminished cerebral substrate delivery. However, defects in the heart-placental axis are expected to further decrease the CPR as placental dysfunction is associated with an increase in UA-PI (i.e., increase in UA resistance). In this setting, placental dysfunction creates a relatively hypoxic fetal environment, and when coupled with altered cardiovascular physiology, this may result in an inadequate cerebrovascular response contributing to abnormal brain development. Indeed, compared to healthy controls, fetuses with CHD have a lower CPR, driven by both a decrease in MCA-PI and an increase in UA-PI (103).

In keeping with this hypothesis, a cohort of fetuses with HLHS, a low CPR $(<1)$ was associated with decreased gray and white matters and decreased surface area of both cerebral hemispheres (35). Furthermore, a nationwide Dutch study found that placental weight in CHD was lower than healthy newborns, especially in TOF, double outlet right ventricular, and major septal defects. Importantly, placental weight was associated with measures of both overall somatic growth and head circumference in fetuses with all subtypes of CHD (104). However, another study did not find an association between placental size and both brain and head volumes in a cohort of 135 fetuses with CHD who underwent fetal MRI in the second and third trimester of pregnancy (21).

\section{Maternal stress}

Prenatal maternal stress has been implicated in adverse pregnancy outcomes and there is a growing considerable interest in its effect on fetal brain growth and neurodevelopmental outcome. Pregnant mothers of fetuses with CHD have been found to experience high incidences of stress, depression, and anxiety. This psychological distress was associated with reduced cerebellar and hippocampal growth during the latter half of gestation when compared to healthy pregnancies (105).

\section{Conclusions}

There is a substantial amount of evidence demonstrating fetuses with CHD, especially severe forms, have structural brain abnormalities during the fetal period across all gestational periods. Factors affecting fetal brain growth in CHD include the genetic background and mismatch between metabolic demand and substrates supplied. However, there is still a need for further investigations aimed to understand the specific contribution of prenatal findings to the overall neurodevelopmental outcome in children with CHD. Indeed, the etiology of brain injury and thus of neurodevelopment impairment in CHD are multifactorial and cumulative both in fetal and postnatal life. Understanding the different etiopathological patterns and their specific contribution to this complex puzzle is a multidisciplinary challenge that must be faced to identify modifiable risk factors and set up preventive and remedial strategies to seek better lifelong neurodevelopmental 
outcomes in children with CHD.

\section{Acknowledgments}

Funding: None.

\section{Footnote}

Provenance and Peer Review: This article was commissioned by the Guest Editor (Antonio F. Corno) for the series "Prenatal Diagnosis in Congenital Heart Defects" published in Translational Pediatrics. The article has undergone external peer review.

Conflicts of Interest: The authors have completed the ICMJE uniform disclosure form (available at http://dx.doi. org/10.21037/tp-20-224). The series "Pre-natal Diagnosis in Congenital Heart Defects" was commissioned by the editorial office without any funding or sponsorship. The authors have no other conflicts of interest to declare.

Ethical Statement: The authors are accountable for all aspects of the work in ensuring that questions related to the accuracy or integrity of any part of the work are appropriately investigated and resolved.

Open Access Statement: This is an Open Access article distributed in accordance with the Creative Commons Attribution-NonCommercial-NoDerivs 4.0 International License (CC BY-NC-ND 4.0), which permits the noncommercial replication and distribution of the article with the strict proviso that no changes or edits are made and the original work is properly cited (including links to both the formal publication through the relevant DOI and the license). See: https://creativecommons.org/licenses/by-nc-nd/4.0/.

\section{References}

1. van der Linde D, Konings EEM, Slager MA, et al. Birth prevalence of congenital heart disease worldwide: A systematic review and meta-analysis. J Am Coll Cardiol 2011;58:2241-7.

2. van der Bom T, Zomer AC, Zwinderman AH, et al. The changing epidemiology of congenital heart disease. Nat Rev Cardiol 2011;8:50-60.

3. Marino BS, Lipkin PH, Newburger JW, et al. Neurodevelopmental outcomes in children with congenital heart disease: Evaluation and management a scientific statement from the american heart association. Circulation 2012;126:1143-72.

4. Peyvandi S, Latal B, Miller SP, et al. The neonatal brain in critical congenital heart disease: Insights and future directions. Neuroimage 2019;185:776-82.

5. Verrall CE, Blue GM, Loughran-Fowlds A, et al. "Big issues" in neurodevelopment for children and adults with congenital heart disease. Open Heart 2019;6:e000998.

6. Gaynor JW, Stopp C, Wypij D, et al. Neuro developmental outcomes after cardiac surgery in infancy. Pediatrics 2015;135:816-25.

7. Licht DJ, Shera DM, Clancy RR, et al. Brain maturation is delayed in infants with complex congenital heart defects. J Thorac Cardiovasc Surg 2009;137:529-36; discussion 536-7.

8. Miller SP, McQuillen PS, Hamrick S, et al. Abnormal brain development in newborns with congenital heart disease. N Engl J Med 2007;357:1928-38.

9. Kelly CJ, Makropoulos A, Cordero-Grande L, et al. Impaired development of the cerebral cortex in infants with congenital heart disease is correlated to reduced cerebral oxygen delivery. Sci Rep 2017;7:15088.

10. Matos SM, Sarmento S, Moreira S, et al. Impact of Fetal Development on Neurocognitive Performance of Adolescents with Cyanotic and Acyanotic Congenital Heart Disease. Congenit Heart Dis 2014;9:373-81.

11. Mulkey SB, Ou X, Ramakrishnaiah RH, et al. White Matter Injury in Newborns with Congenital Heart Disease. Pediatr Neurol 2014;51:377-83.

12. Rosenthal GL. Patterns of prenatal growth among infants with cardiovascular malformations: Possible fetal hemodynamic effects. Am J Epidemiol 1996;143:505-13.

13. Saleem SN. Fetal MRI: An approach to practice: A review. J Adv Res 2014;5:507-23.

14. De Keersmaecker B, Claus F, De Catte L. Imaging the fetal central nervous system. Facts Views Vis Obgyn 2011;3:135-49.

15. Malamateniou C, Malik SJ, Counsell SJ, et al. Motioncompensation techniques in neonatal and fetal MR imaging. Am J Neuroradiol 2013;34:1124-36.

16. Sun L, Macgowan CK, Sled JG, et al. Reduced Fetal Cerebral Oxygen Consumption is Associated With Smaller Brain Size in Fetuses With Congenital Heart Disease. Circulation 2015;131:1313-23.

17. Griffiths PD, Mousa HA, Finney C, et al. An integrated in utero MR method for assessing structural brain abnormalities and measuring intracranial volumes in fetuses with congenital heart disease: results of a 
prospective case-control feasibility study. Neuroradiology 2019;61:603-11.

18. Cercignani M, Bouyagoub S. Brain microstructure by multi-modal MRI: Is the whole greater than the sum of its parts? Neuroimage 2018;182:117-27.

19. Mailath-Pokorny M, Kasprian G, Mitter C, et al. Magnetic resonance methods in fetal neurology. Semin Fetal Neonatal Med 2012;17:278-84.

20. Limperopoulos C, Tworetzky W, McElhinney DB, et al. Brain volume and metabolism in fetuses with congenital heart disease: Evaluation with quantitative magnetic resonance imaging and spectroscopy. Circulation 2010;121:26-33.

21. Andescavage N, Yarish A, Donofrio MT, et al. 3-D volumetric MRI evaluation of the placenta in fetuses with complex congenital heart disease. Placenta 2015;36:1024-30.

22. Masoller N, Sanz-Cortés M, Crispi F, et al. Mid-gestation brain Doppler and head biometry in fetuses with congenital heart disease predict abnormal brain development at birth. Ultrasound Obstet Gynecol 2016;47:65-73.

23. Jørgensen DE, Tabor A, Rode L, et al. Longitudinal brain and body growth in fetuses with and without transposition of the great arteries: Quantitative volumetric magnetic resonance imaging study. Circulation 2018;138:1368-70.

24. Schellen C, Ernst S, Gruber GM, et al. Fetal MRI detects early alterations of brain development in Tetralogy of Fallot. Am J Obstet Gynecol 2015;213:392.e1-392.e7.

25. Krishnan A, Tague L, Rudra H, et al. Clinical course of a fetus with hypoplastic left heart syndrome and premature ductal constriction. Cardiol Young 2019;29:216-8.

26. Al Nafisi B, Van Amerom JF, Forsey J, et al. Fetal circulation in left-sided congenital heart disease measured by cardiovascular magnetic resonance: A case-control study. J Cardiovasc Magn Reson 2013;15:65.

27. Ortinau C, Neil J. The neuroanatomy of prematurity: Normal brain development and the impact of preterm birth. Clin Anat 2015;28:168-83.

28. Puri K, Warshak CR, Habli MA, et al. Fetal somatic growth trajectory differs by type of congenital heart disease. Pediatr Res 2018;83:669-76.

29. Lauridsen MH, Uldbjerg N, Petersen OB, et al. Fetal Heart Defects and Measures of Cerebral Size. J Pediatr 2019;210:146-53.

30. Hahn E, Szwast A, Cnota J, et al. Association between fetal growth, cerebral blood flow and neurodevelopmental outcome in univentricular fetuses. Ultrasound Obstet Gynecol 2016;47:460-5.
31. Hinton RB, Andelfinger G, Sekar P, et al. Prenatal head growth and white matter injury in hypoplastic left heart syndrome. Pediatr Res 2008;64:364-9.

32. Masoller N, Martínez JM, Gómez O, et al. Evidence of second-trimester changes in head biometry and brain perfusion in fetuses with congenital heart disease. Ultrasound Obstet Gynecol 2014;44:182-7.

33. Inversetti A, Fesslova V, Deprest J, et al. Prenatal Growth in Fetuses with Isolated Cyanotic and NonCyanotic Congenital Heart Defects. Fetal Diagn Ther 2020;47:411-9.

34. Abu-Rustum RS, Ziade MF, Abu-Rustum SE, et al. Are There Head Volume Alterations at 11 to 14 Weeks in Fetuses with Congenital Heart Defects? A First Trimester Case Series. AJP Rep 2016;6:e232-e238.

35. Clouchoux C, du Plessis AJ, Bouyssi-Kobar M, et al. Delayed cortical development in fetuses with complex congenital heart disease. Cereb Cortex 2013;23:2932-43.

36. Szymonowicz W, Walker AM, Cussen L, et al. Developmental changes in regional cerebral blood flow in fetal and newborn lambs. Am J Physiol - Hear Circ Physiol 1988;254:H52-8.

37. Pearce $W$. Hypoxic regulation of the fetal cerebral circulation. J Appl Physiol 2006;100:731-8.

38. Olshaker H, Ber R, Hoffman D, et al. Volumetric brain MRI study in fetuses with congenital heart disease. Am J Neuroradiol 2018;39:1164-9.

39. Zeng S, Zhou QC, Zhou JW, et al. Volume of intracranial structures on three-dimensional ultrasound in fetuses with congenital heart disease. Ultrasound Obstet Gynecol 2015;46:174-81.

40. Wong A, Chavez T, O’Neil S, et al. Synchronous Aberrant Cerebellar and Opercular Development in Fetuses and Neonates with Congenital Heart Disease: Correlation with Early Communicative Neurodevelopmental Outcomes, Initial Experience. AJP Rep 2017;7:e17-27.

41. Sanz-Cortes M, Egaña-Ugrinovic G, Zupan R, et al. Brainstem and cerebellar differences and their association with neurobehavior in term small-for-gestational-age fetuses assessed by fetal MRI. Am J Obstet Gynecol 2014;210:452.e1-8.

42. Claessens NHP, Khalili N, Isgum I, et al. Brain and CSF volumes in fetuses and neonates with antenatal diagnosis of critical congenital heart disease: A longitudinal MRI study. Am J Neuroradiol 2019;40:885-91.

43. Luders E, Thompson PM, Narr KL, et al. A curvaturebased approach to estimate local gyrification on the cortical surface. Neuroimage 2006;29:1224-30. 
44. Striedter GF, Srinivasan S, Monuki ES. Cortical Folding: When, Where, How, and Why? Annu Rev Neurosci 2015;38:291-307.

45. Clouchoux C, Kudelski D, Gholipour A, et al. Quantitative in vivo MRI measurement of cortical development in the fetus. Brain Struct Funct 2012;217:127-39.

46. Ortinau CM, Rollins CK, Gholipour A, et al. EarlyEmerging Sulcal Patterns Are Atypical in Fetuses with Congenital Heart Disease. Cereb Cortex 2019;29:3605-16.

47. Peng Q, Zhou Q, Zang M, et al. Reduced fetal brain fissures depth in fetuses with congenital heart diseases. Prenat Diagn 2016;36:1047-53.

48. Mahle WT, Tavani F, Zimmerman RA, et al. An MRI study of neurological injury before and after congenital heart surgery. Circulation 2002;106:I-109-I-114.

49. Mlczoch E, Brugger P, Ulm B, et al. Structural congenital brain disease in congenital heart disease: Results from a fetal MRI program. Eur J Paediatr Neurol 2013;17:153-60.

50. Brossard-Racine M, du Plessis AJ, Vezina G, et al. Prevalence and spectrum of in utero structural brain abnormalities in fetuses with complex congenital heart disease. Am J Neuroradiol 2014;35:1593-9.

51. Bueno D, Parvas M, Garcia-Fernàndez J. The embryonic bloodcerebrospinal fluid barrier function before the formation of the fetal choroid plexus: Role in cerebrospinal fluid formation and homeostasis. Croat Med J 2014;5 5:306-16.

52. Knirsch W, Mayera KN, Scheer I, et al. Structural cerebral abnormalities and neurodevelopmental status in single ventricle congenital heart disease before Fontan procedure. Eur J Cardiothorac Surg 2017;51:740-6.

53. Khalil A, Bennet S, Thilaganathan B, et al. Prevalence of prenatal brain abnormalities in fetuses with congenital heart disease: a systematic review. Ultrasound Obstet Gynecol 2016;48:296-307.

54. Rees S, Harding R, Walker D. An adverse intrauterine environment: implications for injury and altered development of the brain. Int J Dev Neurosci 2008;26:3-11.

55. Woods CG. Human microcephaly. Curr Opin Neurobiol 2004;14:112-7.

56. Barbu D, Mert I, Kruger M, et al. Evidence of fetal central nervous system injury in isolated congenital heart defects: microcephaly at birth. Am J Obstet Gynecol 2009;201:43.e1-7.

57. Shillingford AJ, Ittenbach RF, Marino BS, et al. Aortic morphometry and microcephaly in hypoplastic left heart syndrome. Cardiol Young 2007;17:189-95.
58. Hangge PT, Cnota JF, Woo JG, et al. Microcephaly is associated with early adverse neurologic outcomes in hypoplastic left heart syndrome. Pediatr Res 2013;74:61-7.

59. Glauser TA, Rorke LB, Weinberg PM, et al. Congenital brain anomalies associated with the hypoplastic left heart syndrome. Pediatrics 1990;85:984-90.

60. Evangelou IE, Du Plessis AJ, Vezina G, et al. Elucidating metabolic maturation in the healthy fetal brain using $1 \mathrm{H}-$ MR spectroscopy. Am J Neuroradiol 2016;37:360-6.

61. Sizonenko SV, Camm EJ, Dayer A, et al. Glial responses to neonatal hypoxic-ischemic injury in the rat cerebral cortex. Int J Dev Neurosci 2008;26:37-45.

62. Robertson NJ, Lewis RH, Cowan FM, et al. Early increases in brain myo-inositol measured by proton magnetic resonance spectroscopy in term infants with neonatal encephalopathy. Pediatr Res 2001;50:692-700.

63. O'Donnell LJ, Westin CF. An introduction to diffusion tensor imaging analysis. Neurosurg Clin N Am 2011;22:185-96, viii.

64. Khan S, Rollins CK, Ortinau CM, et al. Tract-specific group analysis in fetal cohorts using in utero diffusion tensor imaging. In: Lecture Notes in Computer Science (including subseries Lecture Notes in Artificial Intelligence and Lecture Notes in Bioinformatics) 2018.

65. Dimitropoulos A, Xu D, Brant R, et al. Brain injury and development in newborns with critical congenital heart disease. Neurology 2013;81:241-8.

66. Lynch JM, Gaynor JW, Licht DJ. Brain injury during transition in the newborn with congenital heart disease: hazards of the preoperative period. Semin Pediatr Neurol 2018;28:60-5.

67. Peyvandi S, Chau V, Guo T, et al. Neonatal brain injury and timing of neurodevelopmental assessment in patients with congenital heart disease. J Am Coll Cardiol 2018;71:1986-96.

68. Claessens NHP, Algra SO, Ouwehand TL, et al. Perioperative neonatal brain injury is associated with worse school-age neurodevelopment in children with critical congenital heart disease. Dev Med Child Neurol 2018;60:1052-8.

69. Wang Y, Cao L, Liang D, et al. Prenatal chromosomal microarray analysis in fetuses with congenital heart disease: a prospective cohort study. Am J Obstet Gynecol 2018;218:244.e1-17.

70. Calcagni G, Unolt M, Digilio MC, et al. Congenital heart disease and genetic syndromes: new insights into molecular mechanisms. Expert Rev Mol Diagn 2017;17:861-70.

71. Fahed AC, Nemer GM. Chapter 6: Genetic Causes of 
Syndromic and Non-Syndromic Congenital Heart Disease Chapter. IntechOpen 2012.

72. Gaynor JW, Gerdes M, Zackai EH, et al. Apolipoprotein E genotype and neurodevelopmental sequelae of infant cardiac surgery. J Thorac Cardiovasc Surg 2003;126:1736-45.

73. Carey AS, Liang L, Edwards J, et al. Effect of copy number variants on outcomes for infants with single ventricle heart defects. Circ Cardiovasc Genet 2013;6:444-51.

74. Homsy J, Zaidi S, Shen Y, et al. De novo mutations in congenital heart disease with neurodevelopmental and other congenital anomalies. Science 2015;350:1262-6.

75. Sifrim A, Hitz MP, Wilsdon A, et al. Distinct genetic architectures for syndromic and nonsyndromic congenital heart defects identified by exome sequencing. Nat Genet 2016;48:1060-5.

76. Rudolph AM. Congenital Diseases of the Heart: ClinicalPhysiological Considerations. Congenital Diseases of the Heart: Clinical-Physiological Considerations 2009:1-538.

77. Rudolph AM. Impaired cerebral development in fetuses with congenital cardiovascular malformations: Is it the result of inadequate glucose supply? Pediatr Res 2016;80:172-7.

78. Koller A, Toth P. Contribution of flow-dependent vasomotor mechanism to the autoregulation of cerebral blood flow. J Vasc Res 2012;49:375-89.

79. Brennand J. Middle cerebral artery Doppler. Australas J Ultrasound Med 2009;12:35-8.

80. Arduini D, Rizzo G. Normal values of pulsatility index from fetal vessels: A cross-sectional study on 1556 healthy fetuses. J Perinat Med 1990;18:165-72.

81. Donofrio MT, Bremer YA, Schieken RM, et al. Autoregulation of cerebral blood flow in fetuses with congenital heart disease: The brain sparing effect. Pediatr Cardiol 2003;24:436-43.

82. Itsukaichi M, Kikuchi A, Yoshihara K, et al. Changes in fetal circulation associated with congenital heart disease and their effects on fetal growth. Fetal Diagn Ther 2011;30:219-24.

83. Williams IA, Fifer C, Jaeggi E, et al. The association of fetal cerebrovascular resistance with early neurodevelopment in single ventricle congenital heart disease. Am Heart J 2013;165:544-50.e1.

84. Zeng S, Zhou J, Peng Q, et al. Assessment by threedimensional power Doppler ultrasound of cerebral blood flow perfusion in fetuses with congenital heart disease. Ultrasound Obstet Gynecol 2015;45:649-56.

85. Kaltman JR, Di H, Tian Z, et al. Impact of congenital heart disease on cerebrovascular blood flow dynamics in the fetus. Ultrasound Obstet Gynecol 2005;25:32-6.

86. Berg C, Gembruch O, Gembruch U, et al. Doppler indices of the middle cerebral artery in fetuses with cardiac defects theoretically associated with impaired cerebral oxygen delivery in utero: Is there a brain-sparing effect? Ultrasound Obstet Gynecol 2009;34:666-72.

87. Yamamoto Y, Khoo NS, Brooks PA, et al. Severe left heart obstruction with retrograde arch flow influences fetal cerebral and placental blood flow. Ultrasound Obstet Gynecol 2013;42:294-9.

88. Turan S, Rosenbloom JI, Hussein M, et al. Longitudinal analysis of head and somatic growth in fetuses with congenital heart defects. J Clin Ultrasound 2017;45:96-104.

89. Kinnear C, Haranal M, Shannon P, et al. Abnormal fetal cerebral and vascular development in hypoplastic left heart syndrome. Prenat Diagn 2019;39:38-44.

90. Man T, He Y, Zhao Y, et al. Cerebrovascular hemodynamics in fetuses with congenital heart disease. Echocardiography 2017;34:1867-71.

91. Szwast A, Tian Z, McCann M, et al. Comparative analysis of cerebrovascular resistance in fetuses with singleventricle congenital heart disease. Ultrasound Obstet Gynecol 2012;40:62-7.

92. Jouannic JM, Benachi A, Bonnet D, et al. Middle cerebral artery Doppler in fetuses with transposition of the great arteries. Ultrasound Obstet Gynecol 2002;20:122-4.

93. Yuen TJ, Silbereis JC, Griveau A, et al. Oligodendrocyteencoded HIF function couples postnatal myelination and white matter angiogenesis. Cell 2014;158:383-96.

94. Marini D, van Amerom J, Saini BS, et al. MR imaging of the fetal heart. J Magn Reson Imaging 2020;51:1030-44.

95. Wheaton WW, Chandel NS. Hypoxia. 2. Hypoxia regulates cellular metabolism. Am J Physiol - Cell Physiol 2011;300:C385-93.

96. Lauridsen MH, Uldbjerg N, Henriksen TB, et al. Cerebral Oxygenation Measurements by Magnetic Resonance Imaging in Fetuses With and Without Heart Defects. Circ Cardiovasc Imaging 2017;10:e006459.

97. You W, Andescavage NN, Kapse K, et al. Hemodynamic responses of the placenta and brain to maternal hyperoxia in fetuses with congenital heart disease by using blood oxygen-level dependent MRI. Radiology 2020;294:141-8.

98. Burton GJ, Jauniaux E. Development of the human placenta and fetal heart: Synergic or independent? Front Physiol 2018;9:373.

99. Linask KK. The heart-placenta axis in the first month of 
pregnancy: Induction and prevention of cardiovascular birth defects. J Pregnancy 2013;2013:320413.

100. Rychik J, Goff D, McKay E, et al. Characterization of the Placenta in the Newborn with Congenital Heart Disease: Distinctions Based on Type of Cardiac Malformation. Pediatr Cardiol 2018;39:1165-71.

101. Saleemuddin A, Tantbirojn P, Sirois K, et al. Obstetric and perinatal complications in placentas with fetal thrombotic vasculopathy. Pediatr Dev Pathol 2010;13:459-64.

102. Jones HN, Olbrych SK, Smith KL, et al. Hypoplastic left heart syndrome is associated with structural and vascular placental abnormalities and leptin dysregulation. Placenta 2015;36:1078-86.

Cite this article as: Lee FT, Seed M, Sun L, Marini D. Fetal brain issues in congenital heart disease. Transl Pediatr 2021;10(8):2182-2196. doi: 10.21037/tp-20-224
103. Arduini M, Rosati P, Caforio L, et al. Cerebral blood flow autoregulation and congenital heart disease: Possible causes of abnormal prenatal neurologic development. J Matern Fetal Neonatal Med 2011;24:1208-11.

104. Matthiesen NB, Henriksen TB, Agergaard P, et al. Congenital heart defects and indices of placental and fetal growth in a nationwide study of 924422 liveborn infants. Circulation 2016;134:1546-56.

105. Wu Y, Kapse K, Jacobs M, et al. Association of maternal psychological distress with in utero brain development in fetuses with congenital heart disease. JAMA Pediatr 2020;174:e195316. 